\title{
Berichte aus den Landesgruppen soweit vorliegend
}

\section{Jahresbericht der Landesgruppe Kärnten 2013}

Die ÖGUM Kärnten besteht aus folgendem Team:

- Vorstand Dr. Gerald Lesnik (Radiologe)

- 1. Stellvertreter Dr Marion Ljuba (Kardiologin)

2. Stellvertreter Dr. Wolfgang Emmer (Internist)

- Kassier Dr. Peter Kuschnig (Radiologe) Insgesamt 5 abgehaltene Kurse im Jahr 2013.

\section{Jahresbericht der Landesgruppe Niederösterreich}

Folgende Veranstaltungen wurden abgehalten:

- 9.11.2013 Kurs: Der geburtshilfliche und gynäkologische Ultraschall.

- 21.11.2013 Fortbildung: Neue genetische Methoden in der Pränataldiagnostik

\section{Jahresbericht der Landesgruppe Oberösterreich}

- 30.01. - 01.02.2013: Aufbaukurs Abdomen; Kursleiter OA Dr. Schneider

- 13.03. - 15.03.2013: Grundkurs Abdomen; Kursleiter OA Dr. Schneider
- 02. 10. - 04.10.2013: $\quad$ Grundkurs Abdomen; Kursleiter OA Dr. Schneider

- 25.04. - 27.04. 2013: Grundkurs Pädiatrische Echokardiografie; Kursleiter Prim. Univ. Doz. Dr. Tulzer

- 07.11. -09.11.2013: Grundkurs Pädiatrische Echokardiografie; Kursleiter Prim. Univ. Doz. Dr. Tulzer

\section{Jahresbericht der Landesgruppe Salzburg}

$\nabla$

- Vorsitz Dr. Erich Hübner, Facharzt für Radiologie, Bischofshofen

- Stellvertreter OA Dr. Christian Weismann, SALK, St.Johanns Spital,

Die Aktivitäten der Salzburger ÖGUMSektion sind auch im Jahr 2012/2013 traditionell durch zahlreiche Kursveranstaltungen gekennzeichnet. Ich darf mich an dieser Stelle erneut sehr herzlich bei den Organisatoren und Referenten dieser ÖGUM-Kurse für die geleistete Fortbildungsarbeit bedanken.

Seit Ende 2009 ist nun die Landesgruppe auch im Internet unter www.oegum-salzburg.at vertreten. Die Seite befindet sich im Aufbau und soll in Zukunft über Kursaktivitäten und Fortbildungen informieren.

Die Landesgruppe Salzburg veranstaltet unter der Leitung des Kursleiters OA. Dr. 
Alois Hollerweger in Zusammenarbeit mit dem $\mathrm{KH}$ der BHB Ultraschallkurse seit März 2010. Ziel dieser Kurse ist es, in Kleingruppen den Teilnehmern eine qualitativ hochwertige Ausbildung zu bieten. Die große Nachfrage sowie die stets ausgebuchten Kurse bestätigen uns in unserer Kursphilosophie. Unverändert im Programm ist ein Kurs welcher sich in zwei Tagen ausführlich mit dem Thema Gastrointestinaltrakt befasst. Auch hier bekundeten die Teilnehmer ein reges Interesse an diesem Thema, so wird auch dieser Kurs, neben einem geplanten Small-Parts/ Gefäßbasiskurs, ein Fixpunkt im „Salzburger Kursgeschehen“.

Insgesamt wurden in 2 Fachbereichen 11

Kurse angeboten im Jahr 2013.

\section{Jahresbericht der Landesgruppe Steiermark \\ $\nabla$}

Insgesamt 14 abgehaltene Kurse im Jahr 2013.

\section{Jahresbericht der Landesgruppe Tirol}

Insgesamt 8 abgehaltene Kurse im Jahr 2013.

\section{Jahresbericht der Landesgruppe} Wien

$\nabla$

- 162 gemeldete Mitglieder

- Vorstand der Landesgruppe Wien (Wiederwahl am 2.12.2011, nächste Wahl für 2014 geplant)

> Vorsitzende Ass. Prof. Dr. Andrea Brichta

> Stellvertreter Prof. Dr. Wolfgang Eppel

> Sekretär Prof. Dr. Dieter Bettelheim

> Kassier Prof. Dr. Wolfgang Dock

5. Mitglied Ass.Prof. Dr. Christian Kollmann

Jahreshauptversammlung: 17.12.13 AKH Wien, Hörsaalzentrum
Abgehaltene Kurse der Landesgruppe Wien: 20 Kurse aus den Bereichen Abdomen (10), Kopf/Hals (3), Bewegungsapparat (3), Gynäkologie (2), Angiologie/Neurologie (1) und Pädiatrie (1)

Fortbildungen der Landesgruppe Wien: Interdisziplinäre Fortbildung (DFP) am 17.12.2013 im AKH Wien, Hörsaalzentrum, verbunden mit der Jahreshauptversammlung der Landesgruppe Wien, organisiert und moderiert von Ass. Prof. Brichta (R. von Hahn/TÜV Süd München: Sicherheit und Risikomanagement bei der Ultraschalldiagnostik (40 Minuten), A. Brichta/Wien: Leberzirrhose im Ultraschall - worauf kommt es an? (30 Minuten)). 